\title{
Motor Inhibition and Learning Impairments in School-Aged Children Following Exposure to Organophosphate Pesticides in Infancy
}

\author{
ORA KOFMAN, ANDREA BERGER, ALI MASSARWA, ALON FRIEDMAN, AND ABED ABU JAFFAR
}

Departments of Behavioral Sciences [O.K., A.B.], Education [A.M.], Neurosurgery and Physiology [A.F.] and Family Medicine [A.A.J.], Zlotowski Center for Neurosciences [O.K., A.B., A.F.], Ben-Gurion University of the Negev, P.O. Box 653, Beersheva, Israel, IL-84105; Clalit Health Services [A.A.J.], P.O.B. 151, Beersheva, Israel, IL-84141

\begin{abstract}
Despite the critical role of acetylcholinesterase (AChE) in cortical function and development, no long-term studies have been conducted in humans on the long-term sequelae of the disruption of the cholinergic system in early childhood. We report a neuropsychological assessment of healthy school-aged children, who had been hospitalized in infancy following exposure to organophosphate pesticides, compared with children exposed to other toxins such as kerosene, and age- and sex-matched non-exposed children. Although overall, the children seem to have overcome the acute one-time exposure incident, and they all attend regular schools, a finer assessment of specific cognitive abilities indicates they are impaired compared with the matched controls. Specifically, the children who had been exposed to organophosphate pesticides had a deficit in inhibitory motor control. Children with pesticide or kerosene poisoning had a retrieval deficit on the acquisition phase of a verbal learning task. (Pediatr Res 60: 88-92, 2006)
\end{abstract}

A cetylcholinesterase inhibitors are the lethal ingredient in organophosphate (OP) and carbamate pesticides. Children in agricultural communities or in urban communities, in which domestic pesticides are used, run the risk of being exposed to acetylcholinesterase inhibitors through hand to mouth contact $(1,2)$. Pesticide poisoning accounts for $15-50 \%$ of the incidents of acute poisoning in children in areas of the world where banned substances are still available $(3,4)$.

In adults, impairment in attention and memory, as well as increased irritability, depression and anxiety have been reported following pesticide poisoning (5). Parents reported a higher incidence of behavioral problems, such as impulsivity and conduct disorder in children 1-2 y after exposure to methyl parathion in their homes (2).

Early childhood is a period of ongoing dendritic growth and synaptogenesis. Huttenlocher and Dabholkar (6) reported that synaptic density peaks at about $3.5 \mathrm{y}$ in the human frontal cortex. Cortical development in toddlers aged $18-24$ mo is

Received September 21, 2005; accepted February 9, 2006.

Correspondence: Ora Kofman, Ph.D., Department of Behavioral Sciences, Ben-Gurion University of the Negev, PO Box 653, Beersheva, Israel IL-84105; Email: kofman@bgu.ac.il

Supported by The National Institute for Psychobiology in Israel grant no. 3-02 to A.B. and O. K.

O.K and A.B made an equal contribution to this study.

DOI: 10.1203/01.pdr.0000219467.47013.35 parallel to that of laboratory rats at around postnatal days $8-20$ (7). The forebrain cholinergic projections to the cortex have been described as "the single most substantial regulatory afferent system of the cerebral cortex" (8). During brain development, acetylcholine ( $\mathrm{ACh})$ and hence its hydrolyzing enzyme, acetylcholinesterase (AChE) and cholinergic projections play major roles in proliferation, migration, and synaptogenesis and the development of normal neural cytoarchitecture (9-11). The effects of administration of OPs during the early postnatal period in laboratory rodents suggested that children exposed to OPs during the period of active synaptogenesis would be particularly vulnerable to cognitive impairment. In rodents, administration of AChE inhibitors in the pre-weaning period led to either increased (12) or decreased (13) locomotion in adults, and an improvement in spatial reference and working memory tasks in females, but a deficit in males (14).

Despite the critical role of both acetylcholine and acetylcholinesterase in cortical development, no long-term studies have been conducted in humans on the sequelae of the disruption of the cholinergic system in early development. We report a neuropsychological assessment of school-aged children who had been hospitalized in infancy following exposure to organophosphate pesticides, compared with children exposed to other toxins, such as kerosene and paint thinner, and age- and sex-matched non-exposed children. A comparison group of children with kerosene poisoning was chosen because this was the most common form of childhood household poisoning in the Negev Bedouin community. It was critical to use a comparison group exposed to poisoning to try to equate factors such as effects of hospitalization and trauma and the reaction of care-takers to a child following an accidental poisoning. Moreover, in retrospective studies of this type, the question of pre-morbid conditions arises. For example, did a mild intellectual or behavioral impairment predispose certain

Abbreviations: AChE, acetylcholinesterase; ADHD, attention deficit hyperactive disorder; $\mathbf{O P}$, organophosphate 
toddlers to accidental self-poisoning? Each group was also compared with a non-exposed age- and sex-matched control group. There has been very limited research on the effects of kerosene-like substances on the developing brain in laboratory animals, in contrast to the above-mentioned research on the long-term effects of exposure to OPs in laboratory mice and rats (12-16). Toluene exposure in rats at postnatal days four through nine increased seizure susceptibility and altered the balance between subunits $2 \mathrm{~A}$ and $2 \mathrm{~B}$ of the NMDA receptor in the cerebellum and hippocampus (17).

\section{METHODS}

This study received human subject approval from the Institutional Review Board for Medical Experimentation in Humans of Ben-Gurion University of the Negev, according to the Helsinki Agreement for Protection of Human Subjects.

Subjects. Fifty-two children participated in this study (32 males). The exposed participants $(n=26)$ were located after screening hospital records of children who were hospitalized for acute poisoning in Soroka Medical Center and treated with standard emergency procedures (18). The study was limited to children aged six to 12 at the time of the study, who were victims of poisoning before age three. All children in this study were native speakers of Arabic from the Bedouin population of the Negev region of Israel. They all attended regular schools.

The examiners were native speakers of Arabic, B.A.- or M.A.-level psychologists who had experience working in the educational psychology service of the Negev Bedouin community. It proved to be very difficult to track the children, based on hospital records from approximately 8 y previously. This was due to the fact that in the years since the poisoning incident, most families had moved, and were not connected to the national phone service. Moreover, since the families dwell in small settlements of large extended families, confidentiality was a major issue in the recruitment process. As a result, the psychologists who administered the tests personally approached the parents and, although they were blind to the type of poisoning of the participant, they knew which children had been exposed.

Among the families that could be traced, based on the information from the time of hospitalization, nine children were exposed to organophosphates and 17 were exposed to kerosene or similar substances such as paint thinner (referred to as the kerosene group). Children from the unexposed control group were recruited through friends and relatives of the exposed children in the same community, matched according to age and sex. The symptoms recorded in the emergency ward were distinct for the OP group and the kerosene group. The distributions of symptoms and demographic data of both groups of exposed children are presented in Table 1.

Neuropsychological evaluation. The neuropsychological testing battery was limited to the areas of memory, attention and inhibitory control, as these behavioral functions have been found to be related to the cholinergic system in animal and human studies (2,14-16). Before the battery was finalized, the first seven children recruited to the study were also tested on the Arabic translation of the WISC-R (19) and compared with age-, sex- and educationmatched unexposed children. The administration of the WISC-R was discontinued in the second phase of the study because it was necessary to reduce the battery to one testing session, due to low compliance of families who were asked to schedule more than one session.

The tests that were selected were translated to Arabic and adapted to the culture of the participants; therefore, no standard norms were used. Instead, the scores of the exposed children were compared with their matched controls.

Long-term verbal memory. Several aspects of memory are tested by this test:

Table 1. Ages and number of children exposed to different toxins and control groups

\begin{tabular}{lccc}
\hline $\begin{array}{c}\text { Substance } \\
\text { exposed }\end{array}$ & $\begin{array}{c}\text { Age (years) } \\
\text { mean (SEM) }\end{array}$ & $\begin{array}{c}\text { Age of } \\
\text { poisoning } \\
\text { mean (SEM) }\end{array}$ & N \\
\hline Organophosphates & $10.30(0.63)$ & $1.96(0.19)$ & 9 \\
Control OP & $9.61(0.47)$ & & 9 \\
Kerosene/thinner & $8.97(0.31)$ & $1.78(0.13)$ & 17 \\
Control kerosene & $8.91(0.31)$ & & 17 \\
\hline
\end{tabular}

1) A list of 15 words (List A) is read to the child, who is asked to recall as many items as possible. This is repeated five times so that a learning curve, marking the increment in number of items recalled is plotted. Aside from the number of items correctly recalled, the examiner makes note of repetitions, which suggest poor monitoring of responses. The list of words was based on an Arabic translation of the word list in the NEPSY (20), with some culture-relevant changes for a desert-dwelling community. For example, the Arabic translation of "policeman" replaced "sailor," and "sand" replaced "movies."

2) After the fifth reading, a different list (List B) is read to the child, who is asked to recall this list. Immediately afterward, the child is asked to recall words from List A. Intrusion errors from List B are noted, and the number of items recalled is compared with the previous performance (Trial 5).

3) Delayed recall of List A is tested after a 20 min delay, during which time the subject completed other unrelated tasks.

4) Recognition memory is tested by reading a list of words which includes all the items from the first and second lists, plus items that are phonetically or semantically similar to words on the first list.

Scoring of the verbal memory test yields several important measures including the learning curve for new material, proactive and retroactive interference (between List A and List B), perseverations and intrusions.

Working memory and attention span: Digit span subtest from the WISC-R. Eight pairs of series ranging from two to nine digits) are read to the child. A score of zero, one, or two is given for number of correct repetitions. For backward digit span, seven pairs of series (two to eight digits long) are used. The child is asked to recite the digits in the reverse order.

Inhibitory control/motor inhibition. Inhibitory control was tested using two standard tests (Statue and Knock Tap from the NEPSY) (20), designed to examine the child's ability to inhibit a prepotent response to one cue and to produce this response in the presence of another cue. In the Statue task, the child is asked to shut his/her eyes for $75 \mathrm{~s}$, with one hand raised as if holding a flag and the other hand resting on a table. The child is instructed not to move or talk until the experimenter says that the time is up. At $15 \mathrm{~s}$ intervals, the experimenter initiates a disruption, such as vocalization, or dropping a pencil. The child is expected to suppress the urge to open his/her eyes, smile, talk, giggle, etc. Each $5 \mathrm{~s}$ interval is scored for movements, eye-opening and vocalizations. In the Knock Tap test, the experimenter sits opposite the child at a table and teaches him to respond to each of the experimenter's hand movements (knocking on the table with a fist, side fist, or flat palm) with a hand movement that is different. For example, if the experimenter taps the table with a flat palm, the child must knock on the table with his/her fist, i.e., suppress the tendency to mimic. The movements are presented sequentially without a break for a total score of 30 . The task is presented in two stages. In the second stage, one of the experimenter's movements acts as a stop signal and the child must not move his hand at all. Points are lost for performing a wrong hand movement, or for moving when the child was supposed to not move at all. Go/no-go tests, in which the subject has to inhibit one motor response and perform another, have been found to be sensitive to frontal lobe damage in adults and are also impaired in children with attention deficit hyperactive disorder (ADHD) (21).

Procedures. Parents were contacted by a research assistant and asked to sign an informed consent letter. Then the children were tested individually in a quiet room. Some of the children from all groups were tested within the local medical clinic in Rahat and the others were tested in their homes. The parents were given a structured interview by the testing psychologist, asking if the child was on medication or had chronic health problems, if they had been hospitalized since the index incident, had repeated a grade, had trouble in any particular subject at school, or had conduct problems in school. In addition, questions concerning the child's pastimes and issues not related to learning problems were included and the child's general demeanor was noted. The interview was not meant to be diagnostic, but was intended to alert us to overt signs of serious developmental delay.

Each child was tested on the same day and in the same place as his matched control. The session length was about $45 \mathrm{~min}$. First they performed trials one through seven of the long-term verbal memory test, followed by the Statue, Knock Tap, Digit Span and Corsi Blocks tests. Last, they performed trials eight and nine of the memory test (Delayed recall of List A, and Recognition). All the participants received a small gift as a token for participation in the study.

Data were analyzed using GLM analysis using STATISTICA (data analysis software system), version 7 (StatSoft, Inc., 2004). All the variables within each domain were analyzed together using ANCOVA, with age as a covariate. For the long-term verbal learning test, ANCOVA was done for the effect of exposure (exposed, control), type of poison (OP or Kerosene) and list presentation (seven trials, including five repeats of the list $\mathrm{A}$, the recall of list $\mathrm{B}$, and the recall of list A following recall of $\mathrm{B}$ ) as a repeated measure. The recognition and delayed memory were analyzed separately using 
ANCOVA for the effects of exposure (exposed, control) and type of poison (OP or Kerosene). Because there were multiple analyses in the memory domain, a Bonferonni correction was used. The attention span tests, Digit Span and Corsi Blocks were analyzed together using ANCOVA for the effects of exposure (exposed, control), type of poison (OP or Kerosene), and order (forward or backward). The motor inhibition tests from the NEPSY were analyzed together using ANCOVA for the effects of exposure (exposed, control), type of poison (OP or Kerosene), and subtest (Knock-Tap or Statue).

\section{RESULTS}

There was no difference between the ages of the exposed children $(9.43 \pm 0.31 \mathrm{y}$, mean \pm SEM) and the matched comparison group $(9.15 \pm 0.26)$, confirming that the matching was appropriate, $F(1,48)=0.82$, n.s. However, there was a difference between of the two subgroups of toxic substances $F(1,48)=5.95, p<0.05$. The mean age and SEM of the children with OP poisoning $(n=9)$ was $9.98 \pm 0.39$ and the mean age and SEM of children who had been poisoned with kerosene $(n=17)$ was $8.94 \pm 0.22$ (Table 1$)$. Age was used as a covariate in the subsequent analyses. There was no difference between the two groups in the age at which the poisoning occurred, $t(23)=0.78, p=0.44$ (Table 1). OP poisoning was confirmed by low serum butyrylcholinesterase activity in all but one child, who was reported to have inhaled insecticide. The mean butyrylcholinesterase level for the children exposed to OP poisoning was (Mean \pm SD) 3,631 \pm 3,701 , and the median value was 1,769 IU/Lit. (normal range 6,100-15,000 IU/Lit).

The structured interview did not reveal any conduct disorders, health or academic problems. One boy who had swallowed paint thinner and one girl who had swallowed kerosene repeated first grade. Academic difficulties in school were not reported by the parents except for two children in each group. None of the children took medication and only one of the children had been hospitalized once, since the poisoning incident. Since this child had also been kept back in first grade and was reported by the parent to have difficulties in school, his performance will be discussed below.

The symptoms of the children derived from the hospital records are summarized in Table 2. Four of the nine children with OP exposure presented with decreased consciousness or stupor, whereas no significant involvement of the CNS was recorded in the files of the children with kerosene poisoning.

The WISC-R scores were compared between the seven children who had been poisoned (three kerosene, four OP) and the matched comparison group. No differences were found between these exposed children and their matched controls, $F(9,171)=0.73$, n.s. The mean (SD) of the full scale IQ was 85.57 (7.54) for the exposed group and 83.57 (6.26) for the comparison group.

Analysis of the verbal memory showed a significant main effect of group, $F(1,47)=9.86, p=0.003,\left(\eta_{\mathrm{p}}{ }^{2}=0.17\right)$, indicating that both groups of children who had been exposed to toxic substances were impaired in verbal learning (Fig. 1). There was an interaction between age and trial, $F(6,282)=$ 2.85, $p<0.01,\left(\eta_{\mathrm{p}}{ }^{2}=0.06\right)$. These effects were significant using a Bonferonni-corrected alpha of 0.017. No other effects were found. No difference between the groups was found for the recognition test, $F(1,47)=0.02, p=0.88$ or the delayedrecall test, $F(1,40)=2.39, p=0.13$ (Note: the sample size for the delayed-recall is smaller, since there were several missing data points due to a technical error). In contrast to the verbal learning test, no differences between the exposed children and controls were found in the Digit Span and Corsi Blocks test.

The effects of poison exposure appeared only in the repeated free recall but not in the delayed or recognition tasks, suggesting that the children did eventually learn the list, even though they had trouble retrieving words in the acquisition phase. To examine the gap between the acquisition phase and the delayed test, post hoc analyses were conducted on difference scores between the acquisition and delayed phases of the test. To analyze the gain in the recall task, the difference between the number of correct words on Trial 5 of the acquisition phase and the number of correct words in the delayed-recall task was compared. Similarly, the gain in recognition was measured as the difference between the number of correct words in the recognition task minus the number of correct words on Trial 5 of the acquisition phase. Lastly, to determine whether there was a specific retrieval deficit, the difference between the delayed recall and recognition task (which also occurred after a 20 min delay) was analyzed. Age was entered as a covariate in each analysis. There was no difference between the control and exposed children in the gain in recall, $F(1,40)=0.09$, n.s. There was a non-significant trend toward a difference between kerosene and OP groups, $F(1,40)=3.54, p=0.07$, such that the children in the OP exposed and control groups had a greater gain in the delayed recall task compared with the children in the kerosene groups. There was no interaction between poisoning status and type of poison, $F(1,40)=0.23$, n.s. In contrast, the recognition gain was significantly greater in the groups exposed to poison compared with the control children, $F(1,47)=5.37, p=0.02$. Moreover, the children in the OP groups (exposed and control) had a significantly greater improvement compared with the children in the kerosene groups (exposed and control), $F(1,47)$ $=5.16, p=0.03$. There was also a significant effect of age, $F(1,47)=22.19, p<0.001$, but as mentioned above, this variable was included as a covariate. There was no interaction between poisoning status and type of poison, $F(1,47)=$ 0.0007 , n.s. The number of words gained on the recognition test compared with Trial 5 was $10.33 \pm 1.9$ for the children exposed to OPs, versus $9.6 \pm 1.4$ for their matched controls

Table 2. Number of children exhibiting each symptom according to records of their hospital intake

\begin{tabular}{ccccccccccccc}
\hline HR $>120$ bps & C & T & SB & F & HT & CC/S & V & D & S & P & MY & Symptom/number of children \\
\hline $1(11)$ & 0 & 0 & $2(22)$ & $2(22)$ & $3(33)$ & $4(44)$ & $5(56)$ & $3(33)$ & $2(22)$ & $1(11)$ & $4(44)$ & OP $(n=9)$ \\
$2(12)$ & $1(6)$ & $1(6)$ & $6(35)$ & 0 & 0 & 0 & $5(29)$ & 0 & 0 & 0 & 0 & Kerosene $(n=17)$ \\
\hline
\end{tabular}

Numbers in parentheses are percentage of total.

MY, myosis; P, perspiration; S, salivation/hypersecretion; D, diarrhea; V, vomiting; CC/S, clouded consciousness or stupor; HT, hypotonia; FS, fasciculation; $\mathrm{SB}$, shortness of breath; T, tearing; C, coughing. 

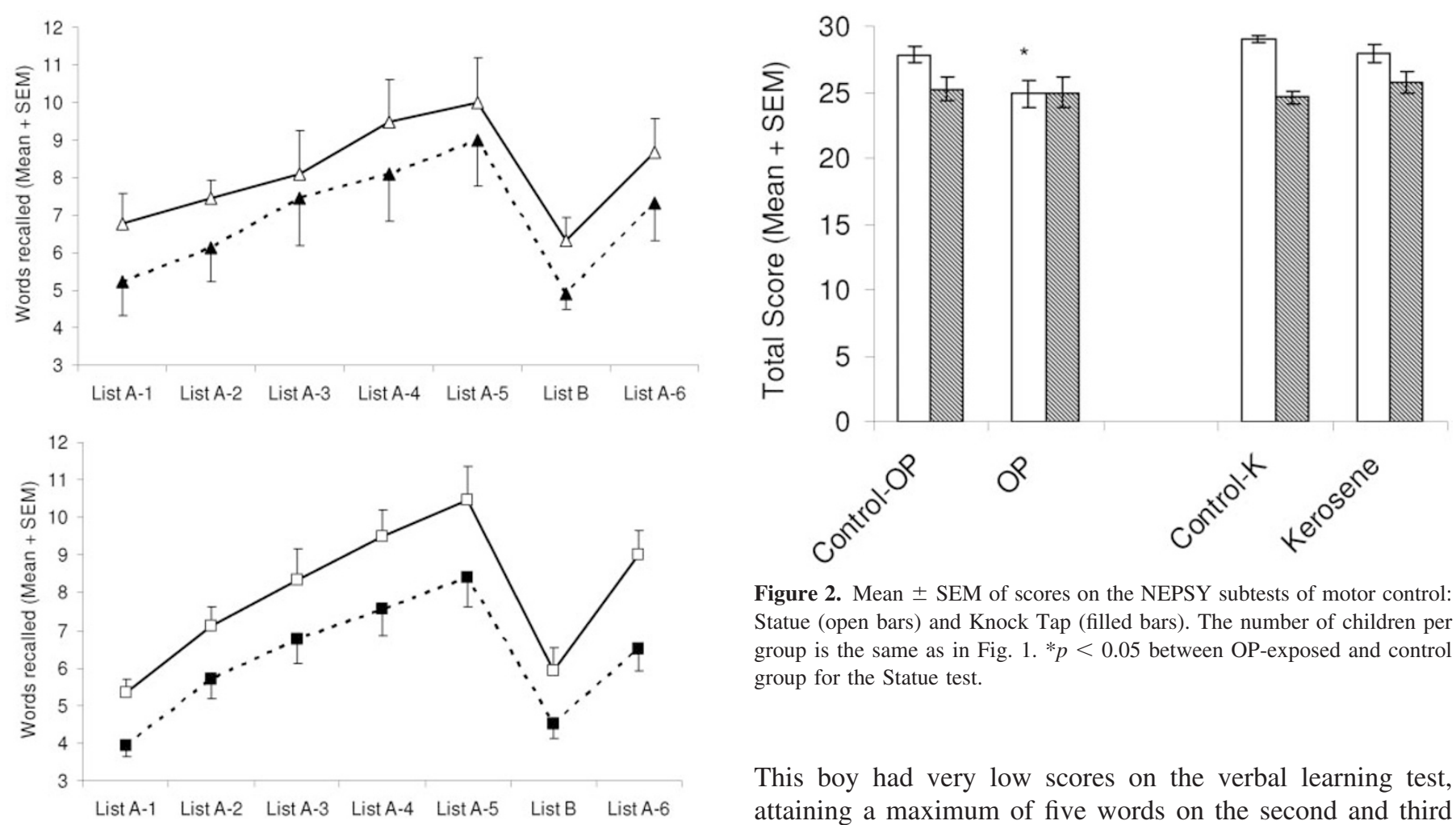

Figure 2. Mean \pm SEM of scores on the NEPSY subtests of motor control: Statue (open bars) and Knock Tap (filled bars). The number of children per group is the same as in Fig. 1. $* p<0.05$ between OP-exposed and control group for the Statue test.

Figure 1. Mean \pm SEM words recalled following presentation of list of 15 words. Children exposed to toxins had lower recall on list A. Top panel, OP exposure ( $n=9$, dashed line) and matched control group ( $n=9$, solid line). Bottom panel, Kerosene exposure ( $n=17$, dashed line) and matched control group $(n=17$, solid line). There is a main effect for exposure to toxins ( $p=$ $0.003)$.

(Mean $\pm \mathrm{SD}$ ). The number of words gained on the recognition test compared with Trial 5 was $8.97 \pm 1.3$ for the children exposed to kerosene, versus $8.9 \pm 1.3$ for their matched controls (Mean $\pm \mathrm{SD}$ ). These two effects are marginally significant using a Bonferonni-corrected alpha of 0.017. There was no evidence of a specific retrieval deficit, as the difference between delayed recall and recognition was similar between the exposed and unexposed groups, $F(1,40)=3.21$, n.s.

Analysis of the inhibitory control battery showed a significant main effect of group, $F(1,47)=8.57, p<0.01,\left(\eta_{\mathrm{p}}{ }^{2}=\right.$ $0.15)$, indicating that the children who were exposed to the toxins were impaired in their motor inhibitory control (Fig. 2). Moreover, there was a main effect of type of poisoning, $F(1,47)=5.09, p<0.05,\left(\eta_{\mathrm{p}}{ }^{2}=0.10\right)$, indicating that the OP subgroup was more impaired than the kerosene subgroup. There was a significant effect of test, $F(1,47)=9.75, p<$ $0.005,\left(\eta_{\mathrm{p}}^{2}=0.17\right)$, indicating that scores were higher in general on the Statue test than on the Knock Tap test. There was also a significant interaction between test and age, $F(1,47)$ $=6.64, p<0.05,\left(\eta_{\mathrm{p}}{ }^{2}=0.12\right)$ which was due to the fact that the older children in the OP poisoned group had lower scores on the Statue test. There was no significant interaction between group, type of poison and test, $F(1,47)=1.94, p=$ 0.17 . As noted above, only one boy who had swallowed paint thinner had a previous hospitalization and repeated first grade.

This boy had very low scores on the verbal learning test, attaining a maximum of five words on the second and third repetitions, but not on the fourth and fifth. He had a perfect score on the Statue test. When the verbal memory was analyzed without this child and his matched control, there was still a significant difference between children exposed to poisoning and unexposed children, $F(1,45)=8.60, p<0.01$, with no difference between OP- and kerosene-exposed children.

\section{DISCUSSION AND CONCLUSIONS}

Despite the fact that incidents of poisoning involving the CNS in infancy and early childhood are not uncommon $(1,2,18)$, there was only one (2) long-term follow-up study after $1-2 \mathrm{y}$. The present study is unique in that the children were followed up several years after a poisoning incident that took place before age three. Children exposed to OPs were compared with children exposed at those ages to kerosene and to matched controls.

Although overall, the children seem to have overcome the acute one-time exposure and they all attend regular schools, a finer assessment of specific cognitive abilities indicates an impairment in the verbal learning and motor inhibition tasks in the exposed compared with matched control children. Further follow-up studies would be required to determine whether these mild developmental delays are long-lasting or if they will be overcome with continued maturation. Four children with OP poisoning showed clouded consciousness upon hospitalization; however, their scores did not differ from the group average on any of the tests.

The Statue test indicates that the children exposed to OPs had more difficult restraining and controlling their motor behavior than the matched controls. This result is in line with previous evidence relating exposure to methyl parathion to a higher incidence of parental report of behavioral problems such as, impulsivity, conduct disorder (2). Impaired motor 
inhibition (21-23) is one of the hallmarks of attention deficit hyperactivity disorder (ADHD), and indeed, the Statue test is included in the "attention" battery of the NEPSY. In laboratory cognitive testing, ADHD subjects do especially poorly in tasks requiring inhibitory control, either motor, i.e., go/no-go stop-signal and, anti-saccade tasks; or cognitive, i.e., Stroop and flanker tasks $(22,23)$.

In the verbal learning test, the difference between the exposed children who were poisoned before age three and controls was found in the rate of learning, but not in delayed recall and recognition. The increased gain in the recognition phase in exposed children suggests that they showed impaired retrieval only in the acquisition phase but not after the delay. This suggests that there was no effect of the early poison exposure on encoding or consolidation. A specific deficit in retrieval, in the absence of encoding and consolidation deficits, is characteristic of lesions in the region of the cholinergic basal forebrain nuclei $(24,25)$. However, this was ruled out by the absence of a significant difference between the delayed recall and recognition scores. The pattern of results suggests a deficit that might be related to fatigability or inability to sustain attention in the learning phase of the study.

While there was no difference in the degree of deficit between the two groups of children who had been exposed to OP or kerosene-like toxins in the memory test, the deficit in motor control was more evident in the OP group in the Statue test. The fact that the deficit was evident only in the Statue test, but not in the Knock Tap test, could be related to the different types of inhibition required by each task. The Knock Tap requires inhibition of a prepotent imitation response to the experimenter's hand movement. On the other hand, the Statue test requires that the child not react to distracting stimuli. One can speculate that this test may be more anxiety-provoking as it is performed with closed eyes. Developmental exposure to acetylcholinesterase inhibitors in mice resulted in less inhibition in anxiety tests such as the elevated plus maze (16).

In summary, this preliminary study of long-term effects of OP poisoning on cognitive behavior in children suggests that subtle behavioral changes may occur, even if they go undetected in school. In view of these findings following a single acute exposure, it would be worthwhile to investigate the contribution of chronic exposure to pesticides, in agricultural communities or fumigated urban housing developments, to childhood behavioral disorders such as ADHD (attention deficit hyperactive disorder).

Although non-specific effects on the CNS, such as hypoxia, metabolic acidosis and/or seizures cannot be conclusively disregarded, these are unlikely to account for the behavioral changes reported. Only one child had a significant hypoxia and metabolic acidosis at admission, and no child in this series was reported to have seizures. The reduced consciousness was probably specifically related to the poison on the brain's cholinergic system (18) although other effects (i.e., on brain paraoxonase) cannot be ruled out at present.

One limitation of this study was the relatively small size of the sample and the fact that the testers were aware which children had been exposed, due to cultural and demographic factors. Other factors, such as family rearing practices and peri- natal complications were not included in the study; however, the fact that the children from the kerosene-exposed and unexposed control groups were chosen from similar backgrounds mitigates the likelihood that these and other uncontrolled factors contributed to the differences between the groups. The current study indicates the importance of conducting long-term follow-up prospective studies of acutely poisoned children. Moreover, research of this kind should be extended to additional populations (1-4) and include a more specific battery of tests focusing on memory and regulation of behavior.

Acknowledgments. The authors thank Amnee Athamneh and Dia Ganaim for testing the children and Oren Tomkins, M.D., M.Sc. for help in collecting the archived data.

\section{REFERENCES}

1. Eskenazi B, Bradman A, Castorina R 1999 Exposures of children to organophosphate pesticides and their potential adverse health effects. Environ Health Persp 107:409-419

2. Ruckart PZ, Kakolweski K, Bove FJ, Kaye W 2004 Long-term neurobehavioral health effects of methyl parathion exposure in children in Misssissippi and Ohio. Environ Health Persp 112:46-51

3. Karalliedde L, Senanayake N 1988 Acute organophosphorus insecticide poisoning in Sri Lanka. Forensic Sci Int 36:97-100

4. Sarker AK, Ghosh S, Barik K 1990 A study of accidental poisoning (in children) in a rural medical college hospital in West Bengal. Indian J Public Health 34:159-162

5. Keifer MC, Mahurin RK 1997 Chronic neurologic effects of pesticide overexposure. Occup Med 12:291-301

6. Huttenlocher PR, Dabholkar AS 1997 Regional differences in synaptogenesis in human cerebral cortex. J Comp Neurol 387:167-178

7. Kolb B 1995 Factors influencing brain plasticity and recovery: Hormones, neuromodulators, neurotrophins and experience. In, Kolb B (ed) Brain Plasticity and Behavior. Lawrence Erlbaum Associates, Mahwah, NJ, pp 133-166

8. Mesulam MM 1994 Structure and function of cholinergic pathways in the cerebral cortex, limbic system, basal ganglia, and thalamus of the human brain. In, Bloom FE, Kupfer DJ (eds) Psychopharmacology: The Fourth Generation of Progress. Raven Press, New York, pp 134-146

9. Dori A, Cohen J, Silverman WF, Pollack Y, Soreq H 2005 Functional manipulations of acetylcholinesterase splice variants highlight alternative splicing contributions to murine neocortical development. Cereb Cortex 15:419-430

10. Hohmann CF 2003 A morphogenetic role for acetylcholine in mouse cerebral neocortex. Neurosci Biobehav Rev 27:351-363

11. Zhu XO, de Permentier PJ, Waite PM 2002 Cholinergic depletion by IgG192saporin retards development of rat barrel cortex. Brain Res Dev Brain Res 136:1-16

12. Ahlbom J, Fredriksson A, Eriksson P 1995 Exposure to an organophosphate (DFP) during a defined period in neonatal life induces permanent changes in brain muscarinic receptors and behavior in adult mice. Brain Res 677:13-19

13. Dam K, Seidler FJ, Slotkin TA 2000 Chlorpyrifos exposure during a critical neonatal period elicits gender-selective deficits in the development of coordination skills and locomotor activity. Brain Res Dev Brain Res 121:179-187

14. Levin ED, Addy N, Nakajima A, Christopher NC, Seidler FJ, Slotkin TA 2001 Persistent behavioral consequences of neonatal chlorpyrifos exposure in rats. Brain Res Dev Brain Res 130:83-89

15. Furey ML, Pietrini P, Haxby JV 2000 Cholinergic enhancement and increased selectivity of perceptual processing during working memory. Science 290:23152319

16. Kofman O, Ben-Bashat G 2006 Diisopropylfluorophosphate administration in the pre-weanling period induces long-term changes in anxiety behavior and passive avoidance in adult mice. Psychopharmacology (Berl) 183:452-461

17. Lee YF, Lo PS, Wang YJ, Hu A, Chen HH 2005 Neonatal toluene exposure alters $\mathrm{N}$-methyl-D-aspartate receptor subunit expression in the hippocampus and cerebellum in juvenile rats. Neuropharmacology 48:195-203

18. Lifshitz M, Shahak E, Bolotin A, Sofer S 1997 Carbamate poisoning in early childhood and in adults. J Toxicol Clin Toxicol 35:25-27

19. Wechlser D 1974 Wechsler Intelligence Scale for Children - Revised. The Psychological Corporation, New York

20. Korkman M, Kirk U, Kemp S 1998 NEPSY. A Developmental Neuropsychological Assessment. The Psychological Corporation, Harcourt Brace and Co., San Antonio

21. Barkley RA 1997 Behavioral inhibition, sustained attention, and executive functions: Constructing a unifying theory of ADHD. Psychol Bull 121:65-94

22. Chae PK, Jung HO, Noh KS 2001 Attention deficit hyperactivity disorder in Korean juvenile delinquents. Adolescence 36:707-725

23. Epstein JN, Goldberg NA, Conners CK, March JS 1997 The effects of anxiety on continuous performance test functioning in an ADHD clinic sample. J Atten Disord 2:45-52

24. Bauer RM, Grande L, Valenstein E 2003 Amnesic disorders. In, Oxford University Press, Oxford, pp 495-573

25. Damasio AR, Graff-Radford NR, Eslinger PJ, Damasio H, Kassell N 1985 Amnesia following basal forebrain lesions. Arch Neurol 42:263-271 